\title{
Development of Pineapple Microsatellite Markers and Germplasm Genetic Diversity Analysis
}

\author{
Suping Feng, ${ }^{1}$ Helin Tong, ${ }^{2}$ You Chen, ${ }^{1}$ Jingyi Wang, ${ }^{2}$ Yeyuan Chen, ${ }^{3}$ Guangming Sun, \\ Junhu $\mathrm{He}^{,}{ }^{3}$ and Yaoting $\mathrm{Wu}^{1}$ \\ ${ }^{1}$ Bioscience and Biotechnology College, Qiongzhou University, Sanya 572200, China \\ ${ }^{2}$ Institute of Tropical Bioscience and Biotechnology, Chinese Academy of Tropical Agricultural Science, Haikou 571101, China \\ ${ }^{3}$ Institute of Tropical Crop Variety Resources, Chinese Academy of Tropical Agricultural Sciences, Danzhou 571737, China \\ ${ }^{4}$ South Subtropical Crops Research Institute, Chinese Academy of Tropical Agricultural Sciences, Zhanjiang 524091, China
}

Correspondence should be addressed to Yaoting Wu; wuyaoting@tsinghua.org.cn

Received 29 April 2013; Accepted 2 July 2013

Academic Editor: Momiao Xiong

Copyright (c) 2013 Suping Feng et al. This is an open access article distributed under the Creative Commons Attribution License, which permits unrestricted use, distribution, and reproduction in any medium, provided the original work is properly cited.

\begin{abstract}
Two methods were used to develop pineapple microsatellite markers. Genomic library-based SSR development: using selectively amplified microsatellite assay, 86 sequences were generated from pineapple genomic library. 91 (96.8\%) of the 94 Simple Sequence Repeat (SSR) loci were dinucleotide repeats (39 AC/GT repeats and $52 \mathrm{GA} / \mathrm{TC}$ repeats, accounting for $42.9 \%$ and $57.1 \%$, resp.), and the other three were mononucleotide repeats. Thirty-six pairs of SSR primers were designed; 24 of them generated clear bands of expected sizes, and 13 of them showed polymorphism. EST-based SSR development: 5659 pineapple EST sequences obtained from NCBI were analyzed; among 1397 nonredundant EST sequences, 843 were found containing 1110 SSR loci (217 of them contained more than one SSR locus). Frequency of SSRs in pineapple EST sequences is $1 S S R / 3.73 \mathrm{~kb}$, and 44 types were found. Mononucleotide, dinucleotide, and trinucleotide repeats dominate, accounting for $95.6 \%$ in total. AG/CT and AGC/GCT were the dominant type of dinucleotide and trinucleotide repeats, accounting for $83.5 \%$ and $24.1 \%$, respectively. Thirty pairs of primers were designed for each of randomly selected 30 sequences; 26 of them generated clear and reproducible bands, and 22 of them showed polymorphism. Eighteen pairs of primers obtained by the one or the other of the two methods above that showed polymorphism were selected to carry out germplasm genetic diversity analysis for 48 breeds of pineapple; similarity coefficients of these breeds were between 0.59 and 1.00, and they can be divided into four groups accordingly. Amplification products of five SSR markers were extracted and sequenced, corresponding repeat loci were found and locus mutations are mainly in copy number of repeats and base mutations in the flanking region.
\end{abstract}

\section{Introduction}

Pineapple (Ananas comosus (L.) Merr.), belonging to Bromeliaceae, ananas, is a perennial evergreen herbaceous fruit tree that produces one of the most famous four tropical fruits beside banana, coconut, and mango. During cultivation and propagation, due to the different naming habits of the propagators and local cultivators, homonym and synonym are very common, nomenclature of pineapple was in chaos, and breeds vary greatly within major groups, which not only hinders rational use of pineapple germplasm resources, but also impedes breeding of better pineapple strains.
Molecular marker technology, such as RFLP, RAPD, and AFLP, has been reported to be used in pineapple germplasm analysis; for example, Duval et al. [1] used RFLP marker in research on germplasm diversity of pineapple. De Fátima Ruas et al. [2] analyzed 18 germplasms of pineapple using RAPD marker and concluded that the cultivated germplasms in this study had a similarity coefficient lower than 0.85 . Duval et al. [3] determined pineapple chloroplast DNA polymorphism using RFLP analysis. Kato et al. [4] analyzed intraspecific DNA polymorphism of pineapple using AFLP assay. Popluechai et al. [5] assessed genetic diversity of nine germplasms of pineapple and divided them into three 
TABle 1: Pineapple materials included in the study.

\begin{tabular}{|c|c|c|c|}
\hline Name & Remarks & Seientifiename & Remarks \\
\hline Sarawak & & $\mathrm{OK}$ & \\
\hline Tainong-6 & Yellow Mauritius $\times$ Cayenne & Unknown & \\
\hline Tainong-20 & & Creanme Pine & \\
\hline Tainong-17 & Cayenne $9 \times$ Rough ${ }^{\top}$ & China Local 2 & \\
\hline Xuli-Tainong & & Common Rough & \\
\hline Japan & & Natal Queen & \\
\hline 2000sh 1 & & Queensland Cayenne & \\
\hline $2000 \operatorname{sh} 2$ & & Riply Queen & \\
\hline Tainong-19 & Cayenneq $\times$ Rough ${ }^{\star}$ & MacGregor & \\
\hline Indonesia Cayenne & & Jin & \\
\hline $\mathrm{HB}$ & & Maroochy & \\
\hline Comte de Paris 1 & & Fresh Premium & \\
\hline Comte de Paris 2 & & Perolera & \\
\hline Boli 1 & 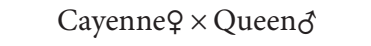 & Alexandria & \\
\hline Thailand THR & & Kallara Local & \\
\hline ST & & Smooth Cayenne 1 & \\
\hline Tainong-18 & & Pattavia & \\
\hline China Local 1 & & Nanglae & \\
\hline Siyuetian & & Smooth cayenne 2 & \\
\hline Hawaii 1 & & New Puket & \\
\hline Red Spanish & & Phuket & \\
\hline Hongpi & & Smooth Cayenne 3 & \\
\hline Tainong-16 & Cayenne $q \times$ Roughơ & Hwaaii 2 & \\
\hline Boli 2 & Cayenneq $\times$ Queen $\widehat{ }{ }^{\top}$ & Tainong-4 & Cayenne $+\underset{\text { Q }}{ } \times$ Queen ${ }^{\top}$ \\
\hline
\end{tabular}

Chinese Academy of Tropic Agricultural Science, Danzhou.

groups based on a 0.77 similarity coefficient. Wöhrmann and Weising [6] developed EST-SSR markers to carry out cross-amplification study within the pineapple bromeliad species, genus, and subfamily. Their results have shown that most genetic markers had low polymorphism, especially when the subjects are closely related. The recently developed microsatellite marker attracts a lot of interests and is being widely used due to its comparatively high polymorphism and genome specificity [7].

SSR markers can be detected by PCR amplification using specific primers which can be developed mainly by classical library screening [8], microsatellite enriching $[9,10], 5^{\prime}$ anchoring PCR technology [11], sequence tagged microsatellite profiling (STMP) [12], selectively amplified microsatellite (SAM) [13], and bioinformatics methods [6, 14, 15]. Among these methods, SAM can generate SSR markers generating multilocus SSR fingerprints, which requires only one pair of primers and has high efficiency in developing informative SSRs. In this study, we designed SSR primers using SAM or bioinformatics method. Those highly informative and reproducible SSR primers were used to carry out germplasm diversity analysis for 48 breeds of pineapples, so as to reveal the genetic relationship among them, provide reference for improvement of the current chaotic situation of pineapple nomenclature, and reveal the regularity of mutation of pineapple SSR loci through amplification, extraction, and sequencing of SSR loci.

\section{Materials and Methods}

2.1. Materials. The Tainong 17 pineapple was used to develop SSR markers; materials for genetic diversity analysis were obtained from Institute of Tropical Crop Variety Resources and South Subtropical Crops Research Institute, Chinese Academy of Tropical Agricultural Sciences (Table 1). DNA was extracted using a modified CTAB method [16]. E. coli strain $\mathrm{DH} 5 \alpha$ for transformation was kept by our laboratory.

2.2. Development of Genomic SSR Markers. Genomic library was constructed in reference to the SAM method [13]. PstI $(15 \mathrm{U} / \mu \mathrm{L}, 0.3 \mu \mathrm{L}), M s e \mathrm{I}(10 \mathrm{U} / \mu \mathrm{L}, 0.5 \mu \mathrm{L}), 10 \mathrm{x}$ NEB buffer II $(5 \mu \mathrm{L})$, and BSA $(10 \mu \mathrm{g} / \mu \mathrm{L}, 0.5 \mu \mathrm{L})$ were added to $1 \mu \mathrm{g}$ of genomic DNA. Reaction was allowed at $37^{\circ} \mathrm{C}$ for $1 \mathrm{~h}$ and terminated by incubation at $65^{\circ} \mathrm{C}$ for $10 \mathrm{~min}$. 5 pmol Pst adaptor and $50 \mathrm{pmol} \mathrm{MseI}$ adaptor were then added and incubated at $45^{\circ} \mathrm{C}$ for $5 \mathrm{~min}$; then T4 DNA ligase $(0.5 \mathrm{U})$, dATP $(100 \mathrm{mM}, 1.8 \mu \mathrm{L})$, and sufficient reaction buffer were added to reach a total volume of $30 \mu \mathrm{L}$, and the system was incubated at $16^{\circ} \mathrm{C}$ for $12 \mathrm{~h}$ for ligation. Product of SAMPCR was separated using denaturing polyacrylamide gel electrophoresis. Based on Hayden and Sharp's [13] work, we increased the number of adaptors, sequences of adaptors, and primers used in this study are shown in Table 2. Target sequences were extracted, cloned, and sequenced, then screened for SSR sequences using the Microsatellite software 
TABLE 2: Sequences of the adapters and primers.

\begin{tabular}{|c|c|}
\hline Name of primers & Sequences of primers $\left(5^{\prime}-3^{\prime}\right)$ \\
\hline \multirow{2}{*}{ PstI adapter } & Sense strand: CTC GGA AGC CTC AGT CCC AGA CTG CGT ACA TGC A-OH \\
\hline & Antisense strand: phos-TGT ACG CAG TCT GGG ACT GAG GCT TCC GAG A-OH \\
\hline \multirow{2}{*}{ MseI adapter } & Sense strand: GAG CAA GGC TCT CAC AAG GAC GAC CGA CGA G-OH \\
\hline & Antisense strand: phos-TAC TCG TCG GTC GTC CTT GTG AGA GCC TTG CT-OH. \\
\hline MseI suppressed amplification primer & GAG CAA GGC TCT CAC A \\
\hline PstI suppression amplification primer & CTC GGA AGC CTC AGT C \\
\hline MseI preamplification primer & GAC GAC CGA CGA GTA AC \\
\hline PstI preamplification primer & AGA CTG CGT ACA TGC AGG A \\
\hline \multirow{12}{*}{ PstI SAM primers } & Adapter 1: AGA CTG CGT ACA TGC AGG ACC \\
\hline & Adapter 2: AGA CTG CGT ACA TGC AGG ACG \\
\hline & Adapter 3: AGA CTG CGT ACA TGC AGG AGC \\
\hline & Adapter 4: AGA CTG CGT ACA TGC AGG A CT \\
\hline & Adapter 5: AGA CTG CGT ACA TGC AGG A TC \\
\hline & Adapter 6: AGA CTG CGT ACA TGC AGG A CA \\
\hline & Adapter 7: AGA CTG CGT ACA TGC AGG A AC \\
\hline & Adapter 8: AGA CTG CGT ACA TGC AGG A GT \\
\hline & Adapter 9: AGA CTG CGT ACA TGC AGG A TG \\
\hline & Adapter 10: AGA CTG CGT ACA TGC AGG A GA \\
\hline & Adapter 11: AGA CTG CGT ACA TGC AGG A AG \\
\hline & Adapter 12: AGA CTG CGT ACA TGC AGG A AT \\
\hline \multirow{4}{*}{$5^{\prime}$-Anchored SSR primers } & PAC: a: KKR YRY YAC ACA CAC ACA C \\
\hline & b: KKY RYR YCA CAC ACA CAC A \\
\hline & PCT: a: KKV RVR VCT CTC TCT CTC T \\
\hline & b: KKR VRV RTC TCT CTC TCT C \\
\hline
\end{tabular}

$\mathrm{K}=\mathrm{G} / \mathrm{T}, \mathrm{R}=\mathrm{G} / \mathrm{A}, \mathrm{Y}=\mathrm{T} / \mathrm{C}, \mathrm{V}=\mathrm{G} / \mathrm{C} / \mathrm{A}, \mathrm{H}=\mathrm{A} / \mathrm{C} / \mathrm{T}$ for $\mathrm{5}^{\prime}$-anchored SSR primer sequences.

(MISA) (http://pgrc.ipk-gatersleben.de/misa/), the criteria for SSR screening were as follows: mononucleotide must be repeated for 10 or more times, dinucleotide and trinucleotide be repeated for six or more times, and $\geq 4$ nucleotide units be repeated for five or more times. Complicated SSRs that are interrupted by no more than 100 bases were also included. Dinucleotide repeats such as AT/TA, CT/AG were regarded as the same type.

Cluster analysis was carried out using stackPACK v 2.2 program [17]. Primers were designed using RIMER5.0 [18] and the main parameters were GC content, $40 \%-60 \%$, annealing temperature, $48-60^{\circ} \mathrm{C}$; anticipated product length, 100-300 bp. The primers were synthesized by Invitrogen.

$20 \mu \mathrm{L}$ PCR reaction system consists of $10 \mathrm{x}$ PCR buffer, $\mathrm{MgCl}_{2}$ (1.5 mM), dNTPs $(0.25 \mathrm{mM})$, forward/backward primers ( 5 pmol each), DNA template (20 ng), and Taq DNA polymerase $(0.15 \mathrm{U})$; reaction program was predenaturation at $94^{\circ} \mathrm{C}$ for $3 \mathrm{~min}, 30$ cycles of denaturation-annealingelongation $\left(94^{\circ} \mathrm{C}\right.$ for $30 \mathrm{sec}, 55^{\circ} \mathrm{C}$ for $45 \mathrm{sec}$, and $72^{\circ} \mathrm{C}$ for $1 \mathrm{~min}$ ), and a final elongation at $72^{\circ} \mathrm{C}$ for $7 \mathrm{~min}$. PCR product was separated using $8 \%$ nondenaturing polyacrylamide gel electrophoresis and visualized by silver staining.

2.3. Development of EST-SSR Markers. ESTs were obtained from the dbEST database of NCBI (http://www.ncbi.nlm .nih.gov/projects/dbEST) registered before February 2012.
ESTs that had PolyT or polyA ( $\geq 5$ repeats) within $50 \mathrm{bp}$ downstream of $5^{\prime}$-end or upstream of $3^{\prime}$-end or shorter than $100 \mathrm{bp}$ were excluded using the EST-trimmer software (http://pgrc.ipk-gatersleben.de/misa/download/est_trimmer .pl); for ESTs that were longer than $700 \mathrm{bp}$, only the first $700 \mathrm{bp}$ at the $5^{\prime}$-end were kept. Then, SSRs were screened using the MISA software. Screening criteria were the same as genome-based development.

Cluster analysis was carried out using stackPACK. Design and synthesis of primers were the same as genome-based development.

2.4. Genetic Diversity Analysis and of Locus Mutation Detection. Eighteen pairs of these newly developed primers that were highly informative and reproducible were selected to carry out genetic diversity analysis for 48 breeds of pineapple. After silver staining, electrophoresis bands were recorded using the Banscan software, for the same migration distance, positive band was recorded as " 1 ," negative band as "0," and failure of amplification as "9." Genetic distance matrix was calculated using NTSYSpc ver 2.1 software (http://www.exetersoftware.com/), evolutionary tree was constructed using the Unweighted Pair Group Method with Arithmetic Mean (UPGMAM) method, primer polymorphism informativeness was calculated using the formula 
TABLE 3: Different units of anchored primer and adapter primer.

\begin{tabular}{lccc}
\hline & PAC & PCT & Total \\
\hline Adapter 1 & 2 & 6 & $\mathbf{8}^{\mathrm{b}}$ \\
Adapter 2 & 3 & 5 & $\mathbf{8}^{\mathrm{b}}$ \\
Adapter 3 & 3 & 4 & $7^{\mathrm{b}}$ \\
Adapter 4 & 1 & 3 & $\mathbf{4}^{\mathrm{b}}$ \\
Adapter 5 & 5 & 6 & $\mathbf{1 1}^{\mathrm{b}}$ \\
Adapter 6 & 3 & 3 & $\mathbf{6}^{\mathrm{b}}$ \\
Adapter 7 & 3 & 3 & $\mathbf{6}^{\mathrm{b}}$ \\
Adapter 8 & 2 & 4 & $\mathbf{6}^{\mathrm{b}}$ \\
Adapter 9 & 1 & 4 & $\mathbf{5}^{\mathrm{b}}$ \\
Adapter 10 & 4 & 4 & $\mathbf{8}^{\mathrm{b}}$ \\
Adapter 11 & 5 & 3 & $\mathbf{8}^{\mathrm{b}}$ \\
Adapter 12 & 3 & 6 & $\mathbf{9}^{\mathrm{b}}$ \\
Total & $\mathbf{3 5}$ & $\mathbf{5 1}^{\mathrm{a}}$ & $\mathbf{8 6}^{\mathrm{a}}$ \\
\hline
\end{tabular}

${ }^{a}$ Number of sequences derived from the units of different PstI adapter and two $5^{\prime}$-anchor primers.

${ }^{\mathrm{b}}$ Number of sequences derived from the units of twelve PstI adapters and different $5^{\prime}$-anchor primers.

${ }^{c}$ Number of sequences derived from the units of twelve PstI adapters and two $5^{\prime}$-anchor primers.

PIC $=1-\sum\left(P_{i}\right)^{2}$, wherein $P_{i}$ stands for the frequency of $i$ th locus in all alleles [21].

Repeat types, (CA) $n$, (GCAGGA) $n$, (AG) $n$, (TCGCAG) $n$, and (TCT) $n$ primers were used to amplify 10 samples that included bands corresponding to all the previous five repeat types; the bands were then recovered, sequenced, and subjected to SSR locus mutation analysis. The ClustalX software was used to compare original sequence and sequencing results.

\section{Results}

3.1. Development of Genomic SSR Markers. Products of SAM PCR were separated using denaturing polyacrylamide gel, and 200-750 bp bands were recovered after silver staining. A total of 99 bands were cloned and sequenced, 86 of them contained SSR loci. Numbers of bands obtained by combination of different anchoring primers and adaptor primers were shown in Table 3. Clustering analysis revealed 68 single sequences, and eight groups of repeated sequences; that is, a total of 76 sequences can be used for primer designing. Pst I SAM primer in combination with $5^{\prime}$ anchoring primer PAC/PGT developed 44 sequences and Pst SAM primer in combination with $5^{\prime}$ anchoring primer PCT/PGA developed 55 sequences, indicating that CT/AG is more abundant than $\mathrm{AC} / \mathrm{GT}$ in pineapple genome (Table 3). All sequences were screened for SSR loci using MISA; 52 GA/CT repeat loci were found and $39 \mathrm{AC} / \mathrm{GT}$ repeat loci were found, which is in accordance with the result developed by different anchoring primers. Three mononucleotide repeats were found and no tri- or more nucleotide repeat locus was found.

Thirty pairs of primers flanking the SSR locus were designed for each of the 36 SSR-containing DNA sequences;

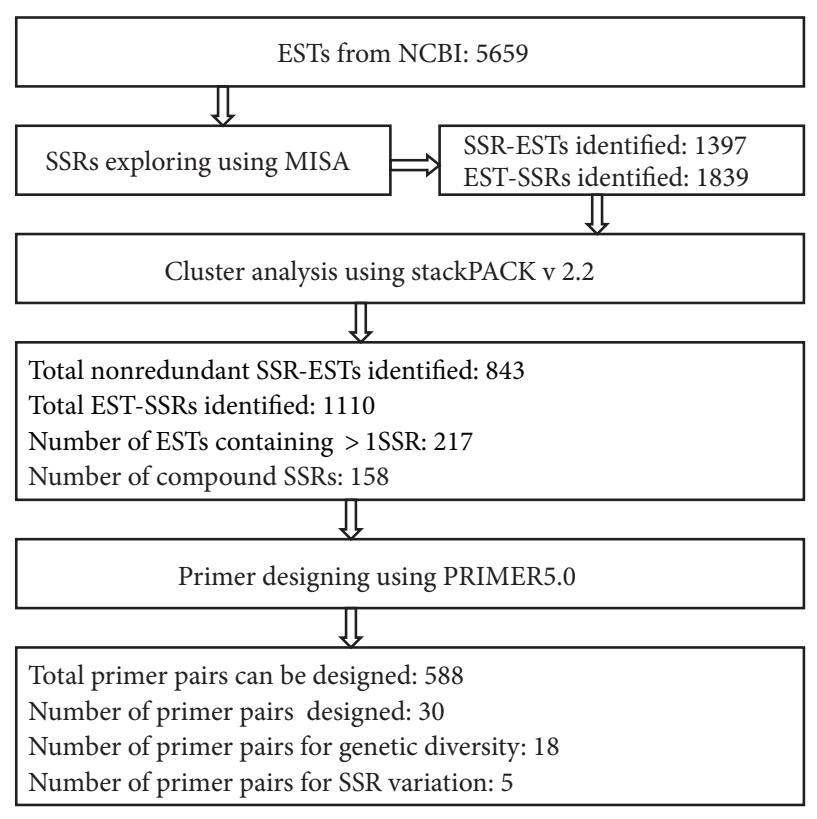

FIGURE 1: Scheme used for data exploring and development of ESTSSRs markers.

24 of them generated clear, reproducible bands of expected size, and 13 of them showed polymorphism when amplifying the selected samples.

3.2. Development of EST-SSR Markers. Fifty-six hundred and fifty-nine EST sequences with a total length of $4,141.084 \mathrm{~kb}$ were downloaded from NCBI database. MISA was used to analyze these sequences and 1397 EST sequences containing 1839 microsatellite loci were developed (Figure 1). Frequency of SSR-containing sequences among all sequences was $24.68 \%$ (one SSR locus every 4.05 ESTs) or one microsatellite locus every $2.25 \mathrm{~kb}$.

Eight hundred and forty-three nonredundant SSRcontaining EST sequences were obtained after cluster analysis on the 1397 EST sequences using stackPACK v 2.2. 620 of them were single sequence and 223 were redundant groups. 1110 SSR loci were identified with MISA, and 217 of these sequences contained more than one SSR locus. Of the 1110 SSR loci, 952 were simple SSRs, and 158 were complicated.

Frequencies of nonredundant EST-SSRs in pineapple ESTs were $1 S S R / 3.73 \mathrm{~kb}$; most of them were small repeating units; taken away mononucleotide repeats, there were 381 (34.3\%) dinucleotide repeats, mostly AG/CT accounting for $83.5 \%$, followed by AT/AT accounting for $10.2 \%$, AC/GT accounted for $6.0 \%$, and CG/CG appeared only once; 158 (14.2\%) trinucleotide repeats were found, mostly AGC/GCT (24.1\%) and AAG/CTT (20.9\%); 23 (2.1\%) 4nucleotide repeats were found, mostly AAAG/CTTT, 12 (1.1\%) 5-nucleotide repeats were found, $33.3 \%$ of them were AAAAG/CTTTT; and 14 (1.3\%) AAAAAG/CTTTTT 6nucleotide repeats were found. In total, 44 types of SSRs were found (Table 4).

Of the 1110 SSR loci identified, taken away the 522 mononucleotide repeats, the other 588 EST-SSRs can be used 
TABLE 4: Frequency and distribution of SSRs in the analysed nonredundant 1110 pineapple ESTs.

\begin{tabular}{|c|c|c|c|c|c|c|c|c|c|c|c|c|}
\hline \multirow{2}{*}{ Repeats motif } & \multicolumn{11}{|c|}{ Number of repeat units } & \multirow{2}{*}{ Total repeats } \\
\hline & 5 & 6 & 7 & 8 & 9 & 10 & 11 & 12 & 14 & 15 & $>16$ & \\
\hline $\mathrm{A} / \mathrm{T}$ & - & - & - & - & - & 149 & 87 & 67 & 35 & 26 & 100 & 511 \\
\hline $\mathrm{C} / \mathrm{G}$ & - & - & - & - & - & 5 & 1 & 1 & 2 & & & 11 \\
\hline $\mathrm{AC} / \mathrm{GT}$ & - & 8 & 4 & 1 & 1 & 2 & 2 & & 1 & & 3 & 23 \\
\hline AG/CT & - & 54 & 34 & 40 & 19 & 25 & 19 & 16 & 19 & 14 & 60 & 318 \\
\hline $\mathrm{AT} / \mathrm{AT}$ & - & 17 & 4 & 9 & 5 & 3 & & 1 & & & & 39 \\
\hline CG/CG & - & & 1 & & & & & & & & & 1 \\
\hline AAC/GTT & - & 2 & 1 & 1 & 2 & & 1 & & 1 & & & 8 \\
\hline AAG/CTT & - & 14 & 8 & 7 & & 2 & 1 & & & & & 33 \\
\hline AAT/ATT & - & 3 & 6 & 1 & 2 & 2 & & & & & & 14 \\
\hline ACC/GGT & - & 1 & 2 & 1 & & 1 & & & & & & 5 \\
\hline ACG/CTG & - & 6 & 6 & 2 & 2 & 1 & 1 & 1 & & & & 19 \\
\hline ACT/ATG & - & 3 & 2 & 1 & & & & 2 & & & & 8 \\
\hline AGC/CGT & - & 10 & 6 & 5 & 6 & 2 & 8 & & & & & 38 \\
\hline AGG/CCT & - & 14 & 8 & 2 & & & & & & & & 24 \\
\hline AGT/ATC & - & & 2 & & & & & & & & & 2 \\
\hline CCG/CGG & - & 3 & 1 & & 2 & 1 & & & & & & 7 \\
\hline AAAC/GTTT & & 1 & & & & & & & & & & 1 \\
\hline AAAG/CTTT & 5 & 2 & 1 & & & & & & & & & 8 \\
\hline AAAT/ATTT & 1 & & & 1 & & & & & & & & 2 \\
\hline AATC/AGTT & 1 & & & & & & & & & & & 1 \\
\hline ACGT/ATGC & & 1 & & & & & & & & & & 1 \\
\hline ACTG/ACTG & & & & & 1 & & & & & & & 1 \\
\hline AGAT/ATCT & 1 & & & & & & & & & & & 1 \\
\hline AGCC/CGGT & & 1 & & & & & & & & & & 1 \\
\hline AGCG/CGCT & 2 & & & & & & & & & & & 2 \\
\hline AGCT/ATCG & 1 & & & & & & & & & & & 1 \\
\hline AGGT/ATCC & & & & 4 & & & & & & & & 4 \\
\hline AAAAG/CTTTT & 3 & 1 & & & & & & & & & & 4 \\
\hline AAAAT/ATTTT & 2 & 1 & & & & & & & & & & 3 \\
\hline AACAC/GTGTT & 1 & & & & & & & & & & & 1 \\
\hline AAGAG/CTCTT & 1 & & & & & & & & & & & 1 \\
\hline AATCG/AGCTT & 1 & & & & & & & & & & & 1 \\
\hline ACCAT/ATGGT & 1 & & & & & & & & & & & 1 \\
\hline AGCCG/CGGCT & 1 & & & & & & & & & & & 1 \\
\hline AAAAAG/CTTTTT & 3 & & & & & & & & & & & 3 \\
\hline AACCCT/ATTGGG & 1 & & & & & & & & & & & 1 \\
\hline AACTAC/ATGTTG & 1 & & & & & & & & & & & 1 \\
\hline AAGAGG/CCTTCT & & 1 & & & & & & & & & & 1 \\
\hline AAGGAG/CCTCTT & 2 & & & & & & & & & & & 2 \\
\hline AAGGCG/CCGCTT & 2 & & & & & & & & & & & 2 \\
\hline AATCCC/AGGGTT & 1 & & & & & & & & & & & 1 \\
\hline ACGGCG/CCGCTG & & 1 & & & & & & & & & & 1 \\
\hline AGCAGG/CCTCGT & 1 & & & & & & & & & & & 1 \\
\hline AGCGTC/AGTCGC & & & 1 & & & & & & & & & 1 \\
\hline
\end{tabular}

for primer designing. Thirty pairs of primers were designed for each of 30 randomly selected EST-SSRs; 26 of them generated clear, reproducible bands, and 22 of them showed polymorphism.
3.3. Genetic Diversity Analysis and Detection of Mutation Locus. Eighteen pairs of highly informative primers developed by EST-SSR or from genomic library were selected to carry out PCR amplification and genetic diversity analysis for 
TABLE 5: Details of the SSR primers for genetic diversity analysis.

\begin{tabular}{|c|c|c|c|c|c|}
\hline $\begin{array}{l}\text { Prime } \\
\text { Pairs }\end{array}$ & $\begin{array}{l}\text { Sequence } \\
\text { ID }\end{array}$ & SSR motif & Forward primer $\left(5^{\prime}-3^{\prime}\right)$ & Reverse primer $\left(5^{\prime}-3^{\prime}\right)$ & $\begin{array}{l}\text { Expected } \\
\text { product } \\
\text { size }(\mathrm{bp})\end{array}$ \\
\hline Bp-01 & AC 1.3 & $(\mathrm{CA}) 8$ & TCACACACACACACACAAAAAC & ATGGATTGCGCTGAGCTG & 119 \\
\hline Bp-08 & AC 6.3 & (TG)8 & ATGATGCCAGTGGAGTGTTC & ACACACACACACTTTTCTCATTG & 152 \\
\hline EP-02 & DT339694 & $(\mathrm{GAA}) 7$ & CGTGCCGCATAAATCAT & TATCTCCTCGCTCCTCTTG & 116 \\
\hline EP-05 & DT339172 & $(\mathrm{CCAT}) 8 \cdots(\mathrm{AT}) 7$ & CAGCCAATAACAACCTCAAG & TCCATACACACAGTACGTCG & 263 \\
\hline EP-06 & DT339094 & (СTTTTT)5 & CGACTCGAGGATTACATTACG & GAGCACAAAGAACCACACAG & 270 \\
\hline EP-09 & DT338799 & (TC)19 & CCGAGGAAGAAGAAGAGGT & GGTCCACAGTTGTTTCAGTT & 160 \\
\hline EP-10 & DT338783 & $(\mathrm{GAT}) 7$ & GACCTTTATCCATCGCATC & CCATCAAACGTGAAATCTTG & 266 \\
\hline EP-11 & DT338752 & $(\mathrm{CAGGAG)5}$ & AGCGAGATAGCAGAGATAGG & TAGAGCGATGTTCGGATG & 180 \\
\hline EP-12 & DT338506 & $(\mathrm{AG}) 6 \cdots(\mathrm{AG}) 6$ & TTAACACATGCACGGAGTAC & CTAAGAGACAACCCAGGAAG & 236 \\
\hline EP-13 & DT338494 & $(\mathrm{CCAT}) 8 \cdots(\mathrm{AT}) 8$ & GCCAATAACAACCTCAAGC & TCCATACACACAGTACGTCG & 263 \\
\hline EP-15 & DT338171 & (GCAGTC)7 & ACCTACAAGTGGTACGTCG & GGAGCAAGGAGTTATTCAG & 242 \\
\hline EP-16 & DT338171 & $(\mathrm{TC}) 6 \cdots(\mathrm{AG}) 18$ & TAGTGAGTCAGGAGGAGAATG & CAAATAAACGGAGCGGAT & 212 \\
\hline EP-20 & DT337383 & $(\mathrm{TCT}) 8$ & TAATCGGGTGGAGTAAGG & GCTCACATAGGCCAATATG & 155 \\
\hline EP-23 & DT337096 & (TC)20 & ATGGTGGTTCACTTATCAGC & AGACATTCAAAGCGGAGAG & 126 \\
\hline EP-24 & DT337054 & $(\mathrm{CT}) 10$ & GCTGCTCTTGCTGCCAT & AAGCCATAGGACCACCAC & 166 \\
\hline EP-26 & DT336292 & $(\mathrm{AT}) 8$ & GAAGCGCAGGTTCGTAAT & ACAGAAGTAGAGGAAAGCAGC & 227 \\
\hline EP-27 & DT336032 & $(\mathrm{TCT}) 6$ & ATACTCTGCTGCTGTGAACG & TTGCACTCCTCTTTGCTAAC & 155 \\
\hline EP-29 & $\mathrm{CO} 731867$ & $(\mathrm{AGC}) 9$ & GCGAGCCTGTTAGACTTTGT & ACGATCTCAGCTGGACCTT & 213 \\
\hline
\end{tabular}

TABLE 6: Comparisons of different search criteria and software for SSR development.

\begin{tabular}{|c|c|c|c|}
\hline & \multicolumn{3}{|c|}{ References } \\
\hline & $\begin{array}{c}\text { Wöhrmann and Weising } \\
{[6]}\end{array}$ & Ong et al. [19] & In this study \\
\hline Software to assemble ESTs & Geneious 5.0 software & SeqMan software & StackPACK v2.2 program \\
\hline Software to search SSRs & SciROKO & SynaRex tool & MISA \\
\hline Search criteria & $\begin{array}{l}\text { Mono- } \geq 15 \text {, di- } \geq 7 \text {, tri-, } \\
\text { tetra-, penta-, hexa- } \geq 5\end{array}$ & Di- $\geq 8$, tri- $\geq 6$, tetra- $\geq 5$ & $\begin{array}{c}\text { Mono- } \geq 10 \text {, di-, tri- } \geq 6 \text {, } \\
\text { tetra-, penta-, hexa- } \geq 5\end{array}$ \\
\hline No. of ESTs analysised & 5659 Moyle et al. [20] & $\begin{array}{l}5931 \text { Moyle et al. [20], Ong } \\
\text { et al. [19] }\end{array}$ & 5659 Moyle et al. [20] \\
\hline No. of SSRs identified & 581 & 416 & 588 \\
\hline No. of SSR motif types & 42 & 5 & 44 \\
\hline Frequency of SSRs & $1 / 4.1 \mathrm{~KB}$ & Not mentioned & $1 / 3.73 \mathrm{~KB}$ \\
\hline SSRs for primerdesign & 537 & 133 & 588 \\
\hline No. of di- & 240 & 203 & 381 \\
\hline No. of tri- & 251 & 213 & 158 \\
\hline No. of (CG)n & 2 & 0 & 1 \\
\hline
\end{tabular}

Mono-, di-, tri-, tetra-, penta- and hexa- represent mononucleotide repeats, dinucleotide repeats, trinucleotide repeats, tetranucleotide repeats and hexanucleotide repeats, respectively.

48 breeds of pineapples (Table 5 ). The results showed that these 48 germplasms of pineapples had similarity coefficients between 0.59 and 1.0. Based on a similarity coefficient of 0.66 , they were divided into four groups: Group 1 containing Sarawak, Tainong-6, Tainong-18, Tainong-19, Comte de Paris 1, Comte de Paris 2, Thailand THR, Kallara local, China Local 1, China Local 2, Phuket, Fresh Premium, New Phuket, Boli 1, Boli 2, Natal Queen, Seiyuetian, OK, Tainong16, Tainong-4, Xuli Tainong, MacGregor, Common Rough,
Alexandria and Ripley Queen; Group 2 containing Tainong20, 2000sh 1, Indonesia cayenne, Hongpi, Unknown, Hawaii 1, Smooth cayenne 1, Smooth cayenne 2, Creanme pine, Smooth cayenne 3, Pattavia, Nanglae, Japan, HB, Maroochy, Tainong-17, Perolera, Hawaii 2, ST, and Queensland Cayenne; Group 3 containing 2000sh 2 and Jin; and Group 4 containing only Red Spanish (Figure 2).

Five pairs of primers were used for recovery and sequencing, of which four were developed from EST-SSR, and the 


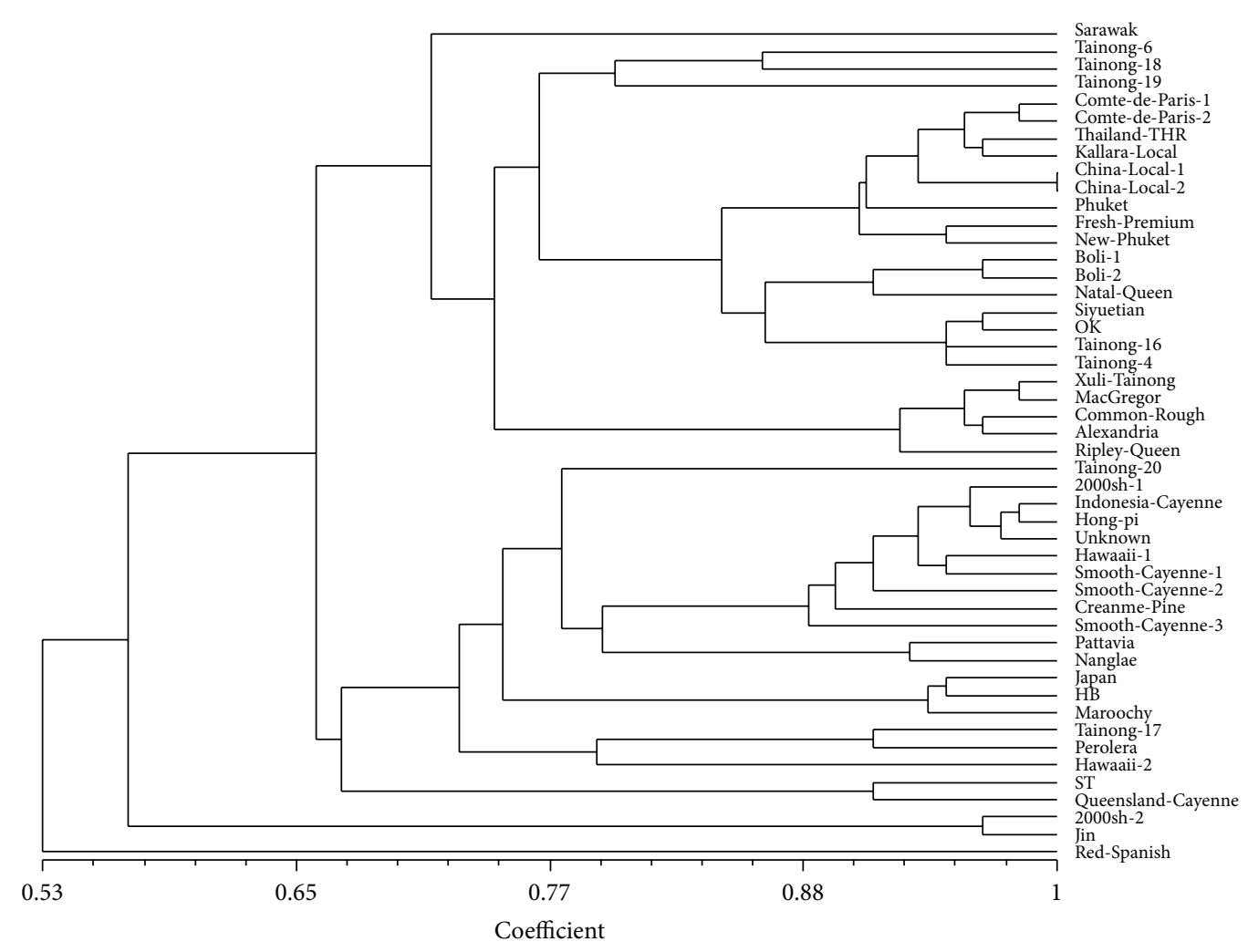

FIGURE 2: Dendrogram of pineapple varieties based on 18 SSRs primer pairs.

other one was developed from genomic library. After PCR amplification and sequencing, these five pairs of primers generated 73 sequences, different SSR markers generated corresponding sequences after amplification and sequencing. Through comparison by ClustalX software, insertions, deletions, transversions, and conversions of these SSR loci and flanking sequences were revealed (Figure 3 ).

\section{Discussion}

4.1. Efficiency of SAM Method to Develop SSR Markers. This study used the SAM method invented by Hayden and Sharp [13] to develop positive clones from pineapple genome for sequencing. Ninety nine clones were sequenced and 86 of them contained 94 SSR loci. Thirty-six of these sequences were selected, and 36 pairs of primers flanking the SSR loci were designed, one for each, and 24 of them generated clear and reproducible bands of expected size, and 13 of them showed polymorphism when amplifying selected samples. $86.9 \%$ of all sequenced clones were positive, and frequency of SSR marker showing polymorphism was $13.1 \%$, which is lower than [22] results for rubber trees (24.6\%) and Wang et al. [23] for banana (19.5\%). This may be due to variations between different materials; although SSRs are widely distributed in eukaryotic genomes, their content, type, and copy number vary between different materials. Even within the same species, there would also be variances. Another modification was that primers were designed on repeating sequences of microsatellite, and only a portion of flanking sequence was used instead of the whole initial $5^{\prime}$ anchoring primer. This may have elevated reproducibility of the primers but may lower their polymorphism. Comparatively, the SAM method is much more efficient in developing SSRs than conventional constructing and screening from genomic library of small inserts or STMS method. For example, Ujino et al. [8] acquired only three positive SSR-containing sequences out of 6000 clones $(0.05 \%)$ using conventional method, and Rajora et al. [24] developed 71 positive clones out of 4028 (1.8\%) using STMS method.

4.2. SSR Sequence Analysis. All sequences were screened for repeat loci using MISA, AC/GT repeats accounted for $41.5 \%$, GA/TC repeats accounted for $55.3 \%$, which was in consistence with results acquired by different anchoring primers. Mononucleotide A/T repeats occurred three times (3.2\%) no $\geq 3$ nucleotide repeats were found. Relatively fewer repeat types were obtained in comparison with Rivera et al. [25], Viruel and Hormaza [26]. Such phenomenon can be explained by the following facts: first, the choice of length and type of the additional $3^{\prime}$ bases of preamplification primers reduced SSR productivity at the same time of reducing complexity of the template [13]; second, the parameters set for repeat screening also have certain effects; for example, we have set that 6-nucleotide must repeat five or more times and there was no such loci, but if the parameter was changed to four or more times, there would be one CAAACA/TGTTTG repeat; third, choice of probes may influence frequency of corresponding repeats; for example, Rajora et al. [24] used 


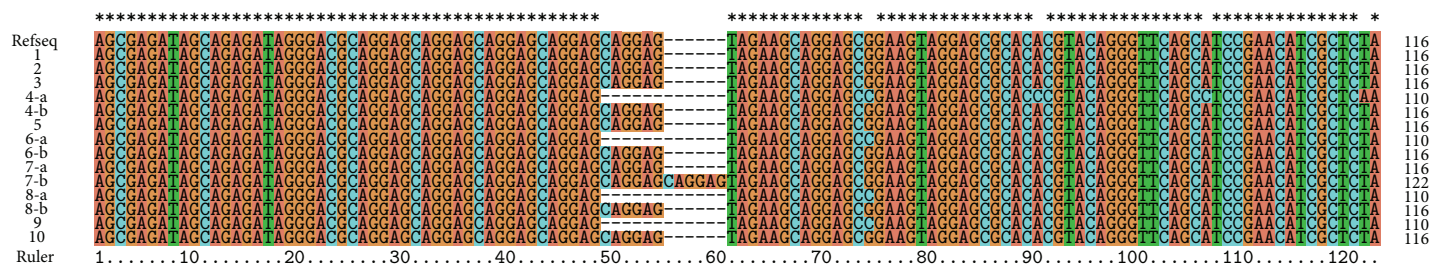

(a)

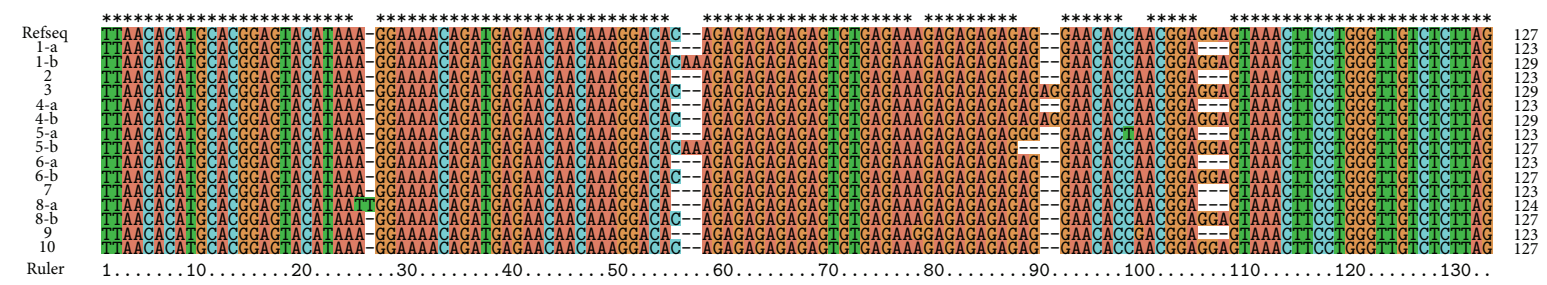

(b)

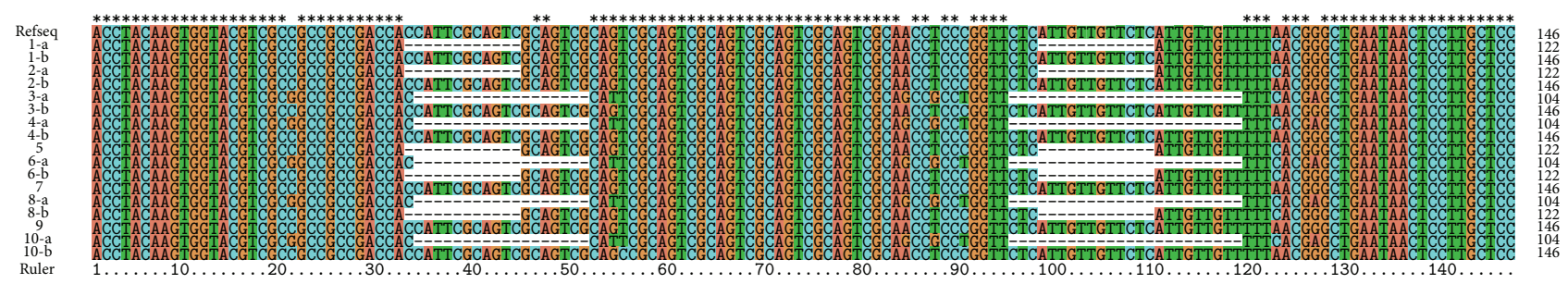

(c)

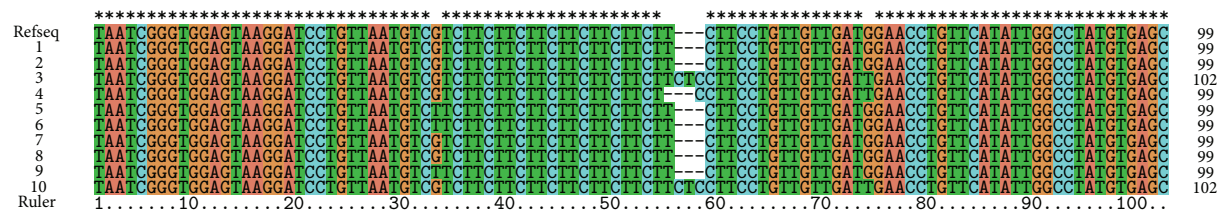

(d)

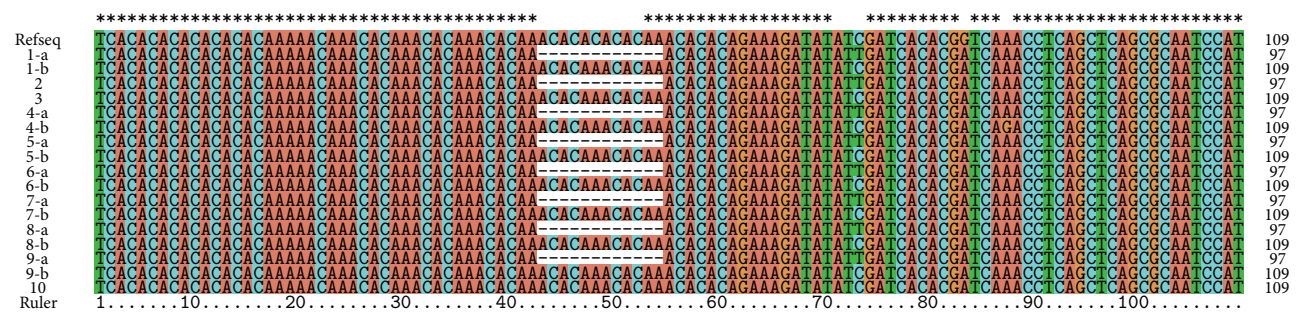

(e)

FIGURE 3: Sequences obtained using five SSRs markers amplifying across Ananas comosus. (a), (b), (c), (d), and (e) represent the marker EP11, EP-12, EP-15, EP-20, and Bp-01, respectively. RefESTs in (a), (b), (c), and (d) represent the accession numbers: DT338752.1, DT338506.1, DT338171.1, and DT337383.1 in NCBI database, respectively. (e) represents AC1.3. The suffixes "a" and "b" represent the allele numbers. 1-10 represent: 1-Tainong-17, 2-Japan, 3-Comte de Paris, 4-Tainong-18, 5-Red Spanish, 6-MacGregor, 7-Jin, 8-Alexandria, 9-Pattavia; and 10-Phuket.

oligonucleotide sequences corresponding to different SSRs and the resulted SSRs had similar repeat units to the probes.

\subsection{Comparison of the Development of Genomic SSR and EST-} $S S R$. EST-SSR marker has unique advantages [27], including being able to detect polymorphism of expressing regions of the genome, high versatility, and relatively low development cost. Thus, it is of great value in genetic mapping, diversity of genetic resources, discovery, and positioning of functional genes, researches on origin of species, evolution, and genomic comparison [28].

Wöhrmann and Weising [6] screened NCBI database for SSRs, setting the criteria as no less than 15 times for mononucleotide repeats; no less than seven times for dinucleotide repeats, and no less than five times for 3-6 nucleotide repeats. Forty-two types SSRs were revealed from 5659 ESTs; one 
SSR occurred every $4.1 \mathrm{~kb}$ on average. Trinucleotide repeats was the most common, followed by dinucleotide repeats. Ong et al. [19] also developed SSR markers from 5931 ESTs using SynaRex tool. To ensure comparability between ESTbased and genome-based SSR development, different analytic software and the same criteria for SSR screening were used in this study. For EST-based SSR marker development, due to differences in size of database, criteria for SSR screening, and tools for SSR development, the distribution, frequency, and abundance of SSRs also vary (Table 6), as concluded by Varshney et al. [28].

In this study, the rate of polymorphic SSR marker was $13.1 \%$ for SAM-developed SSRs, and 2.1\% for EST-developed SSRs, showing a higher efficiency of SSR development by SAM method than genomic library-based method which was in consistence with results reported for soybean $[29,30]$ and rubber trees $[22,31]$.

\subsection{Genetic Diversity Analysis for Pineapple Germplasms} Using SSR Markers. In the present study, 48 germplasms of pineapple were divided into four subgroups, instead of three (Caine, Queen, and Spain) by conventional morphological classification. It can be observed from the cluster analysis, of the 25 germplasms in the first group, Kallara local, Phuket, New Phuket, Natal Queen, MacGregor, Common Rough, Alexandria and Riply Queen, and so forth, belong to the Queen group, and the others such as Tainong-6, Tainong18, Tainong-19, Boli 1, Boli 2, Tainong-16 and Tainong-4 were the hybridizations of Caine and Queen, and so forth. The germplasms in the second group are morphologically divided into the Caine group, while Indonesia cayenne, Hawaii 1, Smooth cayenne 1, Smooth cayenne 2, Smooth cayenne 3, Pattavia, Nanglae, Hawaii 2, and Queensland Cayenne, and so forth, are hybridizations of Caine and Queen; the fourth group only contains the Red Spanish germplasm of the Spain group.

Some taxonomists regarded Perolera as a new breed, and in this study, it was clustered into the second group, which is closely related to the Caine group. The Sarawak germplasm was thought to belong to the Caine group, but it was actually clustered into the Queen group. This phenomenon may be due to non-unified classification standards for pineapple that leads to different classification; the name for the germplasm's confusion, for exemple, one germplasm has multiple names or a single name used by multiple germplasms because of frequent regional and international exchange of pineapple germplasms; the internal limitation of morphological classification that characteristics of a germplasm is easily affected by environmental conditions. During cultivation and propagation, due to different naming habits of the propagators and local cultivators, homonym and synonym are very common, nomenclature of pineapple was in chaos, and germplasms vary greatly within major groups. In addition, SSR reveals not only genetic variations at the DNA level, but also differences in genotype between germplasms. Genome DNA contains not only structural genes, but also some silence genes which had yet no clear function, and the perceptible phenotype is the results of functional gene expression under influence of both internal and external environment. So, difference in DNA structure may not necessarily lead to differences in morphology.

4.5. Analysis of SSR Mutation. Mutation of SSR mainly came from base changes in the flanking sequence and repeat region. In this study, we found no insertion or deletion mutations at the EP-11 or EP-20 loci, at the EP-12 locus, Alexandria-a, Tainong-17-b, and Red Spanish-b had " $T$ " and "AA" insertion, respectively; flanking sequence of $\mathrm{Bp}$ and $\mathrm{EP}$ had deletions (Figure 3).

Flanking sequence of corn had insertion mutations [32]. Gutierrez et al. [33] found in their research on M. truncatula that sequence variation was mainly due to variation in copy number of repeats of the SSR region, as well as insertion, deletion, and nucleotide substitution mutations. Symonds and Lloyd [34] pointed out that interruptions in the SSR region shortened the SSR sequence; in this study, it was observed that nucleotide substitution resulted in decrease in copy number of repeats; a single long repeat sequence was divided into several smaller repeat sequences or became shorter. For example, the Golden pineapple had a CAGGAG insertion at the EP-11 " $\mathrm{b}$ " locus, increasing repeat number; the "T" of EP-20, Tainong-18 sequence was replaced with " $\mathrm{C}$," leading to decrease in TCT repeats; Red Spanish-b had its " $\mathrm{A}$ " replaced with "G" at the EP-12 locus and was thus divided into smaller repeating units.

\section{Conflict of Interests}

The authors declare that there is no conflict of interest with MicroSAtellite software (MISA) (http://pgrc.ipk-gatersleben .de/misa/), EST-trimmer software (http://pgrc.ipkgatersleben.de/misa/download/est_trimmer.pl), and other commercial identities in this paper.

\section{Authors' Contribution}

Suping Feng and Helin Tong contributed equally to this research.

\section{Acknowledgment}

The present work was supported by Main Programme of National Science Infrastructure Platform (2005DKA21000-543).

\section{References}

[1] M. F. Duval, J. L. Noyer, X. Perrier, C. D’Eeckenbrugge, and P. Hamon, "Molecular diversity in pineapple assessed by RFLP markers," Theoretical and Applied Genetics, vol. 102, no. 1, pp. 83-90, 2001.

[2] C. De Fátima Ruas, P. M. Ruas, and J. R. S. Cabral, "Assessment of genetic relatedness of the genera Ananas and Pseudananas confirmed by RAPD markers," Euphytica, vol. 119, no. 3, pp. 245252, 2001. 
[3] M. F. Duval, G. S. C. Buso, F. R. Ferreira et al., "Relationships in Ananas and other related genera using chloroplast DNA restriction site variation," Genome, vol. 46, no. 6, pp. 990-1004, 2003.

[4] C. Y. Kato, C. Nagai, P. H. Moore et al., "Intra-specific DNA polymorphism in pineapple (Ananas comosus (L.) Merr.) assessed by AFLP markers," Genetic Resources and Crop Evolution, vol. 51, no. 8, pp. 815-825, 2005.

[5] S. Popluechai, S. Onto, and P. D. Eungwanichayapant, "Relationships between some Thai cultivars of pineapple (Ananas comosus) revealed by RAPD analysis," Songklanakarin Journal of Science and Technology, vol. 29, no. 6, pp. 1491-1497, 2007.

[6] T. Wöhrmann and K. Weising, "In silico mining for simple sequence repeat loci in a pineapple expressed sequence tag database and cross-species amplification of EST-SSR markers across Bromeliaceae," Theoretical and Applied Genetics, vol. 123, no. 4, pp. 635-647, 2011.

[7] M. J. Kinsuat and S. V. Kumar, "Polymorphic microsatellite and cryptic simple repeat sequence markers in pineapples (Ananas comosus var. comosus)," Molecular Ecology Notes, vol. 7, no. 6, pp. 1032-1035, 2007.

[8] T. Ujino, T. Kawahara, Y. Tsumura, T. Nagamitsu, H. Yoshimaru, and W. Ratnam, "Development and polymorphism of simple sequence repeat DNA markers for Shorea curtisii and other Dipterocarpaceae species," Heredity, vol. 81, no. 4, pp. 422-428, 1998.

[9] H.-B. Huang, Y.-Q. Song, M. Hsei et al., "Development and characterization of genetic mapping resources for the turkey (Meleagris gallopavo)," Journal of Heredity, vol. 90, no. 1, pp. 240-242, 1999.

[10] S. N. Nayak, H. Zhu, N. Varghese et al., "Integration of novel SSR and gene-based SNP marker loci in the chickpea genetic map and establishment of new anchor points with Medicago truncatula genome," Theoretical and Applied Genetics, vol. 120, no. 7, pp. 1415-1441, 2010.

[11] P. J. Fisher, R. C. Gardner, and T. E. Richardson, "Single locus microsatellites isolated using $5^{\prime}$ anchored PCR," Nucleic Acids Research, vol. 24, no. 21, pp. 4369-4371, 1996.

[12] M. J. Hayden and P. J. Sharp, "Sequence-tagged microsatellite profiling (STMP): a rapid technique for developing SSR markers," Nucleic Acids Research, vol. 29, no. 8, article e43, 2001.

[13] M. J. Hayden and P. J. Sharp, "Targeted development of informative microsatellite (SSR) markers," Nucleic Acids Research, vol. 29, no. 8, article e44, 2001.

[14] L. Ramsay, M. Macaulay, S. Degli Ivanissevich et al., "A simple sequence repeat-based linkage map of Barley," Genetics, vol. 156, no. 4, pp. 1997-2005, 2000.

[15] L. F. Gao, J. F. Tang, H. W. Li, and J. Z. Jia, "Analysis of microsatellites in major crops assessed by computational and experimental approaches," Molecular Breeding, vol. 12, no. 3, pp. 245-261, 2003.

[16] S. Porebski, L. G. Bailey, and B. R. Baum, "Modification of a CTAB DNA extraction protocol for plants containing high polysaccharide and polyphenol components," Plant Molecular Biology Reporter, vol. 15, no. 1, pp. 8-15, 1997.

[17] R. T. Miller, A. G. Christoffels, C. Gopalakrishnan et al., "A comprehensive approach to clustering of expressed human gene sequence: the Sequence Tag Alignment and Consensus
Knowledge base," Genome Research, vol. 9, no. 11, pp. 1143-1155, 1999.

[18] J. G. Wetmur, "DNA Probes: applications of the principles of nucleic acid hybridization," Critical Reviews in Biochemistry and Molecular Biology, vol. 26, pp. 227-259, 1991.

[19] W. D. Ong, C. L. Y. Voo, and S. V. Kumar, "Development of ESTs and data mining of pineapple EST-SSRs," Molecular Biology Reports, vol. 39, pp. 5889-5896, 2012.

[20] R. L. Moyle, M. L. Crowe, J. Ripi-Koia, D. J. Fairbairn, and J. R. Botella, "PineappleDB: an online pineapple bioinformatics resource," BMC Plant Biology, vol. 5, article 21, 2005.

[21] D. Botstein, R. L. White, M. Skolnick, and R. W. Davis, "Construction of a genetic linkage map in man using restriction fragment length polymorphisms," American Journal of Human Genetics, vol. 32, no. 3, pp. 314-331, 1980.

[22] F. Yu, B.-H. Wang, S.-P. Feng, J.-Y. Wang, W.-G. Li, and Y.-T. $\mathrm{Wu}$, "Development, characterization, and cross-species/genera transferability of SSR markers for rubber tree (Hevea brasiliensis)," Plant Cell Reports, vol. 30, no. 3, pp. 335-344, 2011.

[23] J. Y. Wang, L. S. Zheng, B. Z. Huang, W. L. Liu, and Y. T. $\mathrm{Wu}$, "Development, characterization, and variability analysis of microsatellites from a commerical cultivar of Musa acuminate," Genetic Resources and Crop Evolution, vol. 57, pp. 553-563, 2010.

[24] O. P. Rajora, M. H. Rahman, S. Dayanandan, and A. Mosseler, "Isolation, characterization, inheritance and linkage of microsatellite DNA markers in white spruce (Picea glauca) and their usefulness in other spruce species," Molecular and General Genetics, vol. 264, no. 6, pp. 871-882, 2001.

[25] R. Rivera, K. J. Edwards, J. H. A. Barker et al., "Isolation and characterization of polymorphic microsatellites in Cocos nucifera L," Genome, vol. 42, no. 4, pp. 668-675, 1999.

[26] M. A. Viruel and J. I. Hormaza, “Development, characterization and variability analysis of microsatellites in lychee (Litchi chinensis Sonn., Sapindaceae)," Theoretical and Applied Genetics, vol. 108, no. 5, pp. 896-902, 2004.

[27] P. K. Gupta, S. Rustgi, S. Sharma, R. Singh, N. Kumar, and H. S. Balyan, "Transferable EST-SSR markers for the study of polymorphism and genetic diversity in bread wheat," Molecular Genetics and Genomics, vol. 270, no. 4, pp. 315-323, 2003.

[28] R. K. Varshney, A. Graner, and M. E. Sorrells, "Genic microsatellite markers in plants: features and applications," Trends in Biotechnology, vol. 23, no. 1, pp. 48-55, 2005.

[29] J. Rongwen, M. S. Akkaya, A. A. Bhagwat, U. Lavi, and P. B. Cregan, "The use of microsatellite DNA markers for soybean genotype identification," Theoretical and Applied Genetics, vol. 90, no. 1, pp. 43-48, 1995.

[30] Q. J. Song, L. F. Marek, R. C. Shoemaker et al., "A new integrated genetic linkage map of the soybean," Theoretical and Applied Genetics, vol. 109, no. 1, pp. 122-128, 2004.

[31] S. P. Feng, W. G. Li, H. S. Huang, J. Y. Wang, and Y. T. $\mathrm{Wu}$, "Development, characterization and cross-species/genera transferability of EST-SSR markers for rubber tree (Hevea brasiliensis)," Molecular Breeding, vol. 23, no. 1, pp. 85-97, 2009.

[32] Y. Matsuoka, S. E. Mitchell, S. Kresovich, M. Goodman, and J. Doebley, "Microsatellites in Zea-variability, patterns of mutations, and use for evolutionary studies," Theoretical and Applied Genetics, vol. 104, no. 2-3, pp. 436-450, 2002. 
[33] M. V. Gutierrez, M. C. Vaz Patto, T. Huguet, J. I. Cubero, M. T. Moreno, and A. M. Torres, "Cross-species amplification of Medicago truncatula microsatellites across three major pulse crops," Theoretical and Applied Genetics, vol. 110, no. 7, pp. 12101217, 2005.

[34] V. V. Symonds and A. M. Lloyd, "An analysis of microsatellite loci in Arabidopsis thaliana: mutational dynamics and application," Genetics, vol. 165, no. 3, pp. 1475-1488, 2003. 

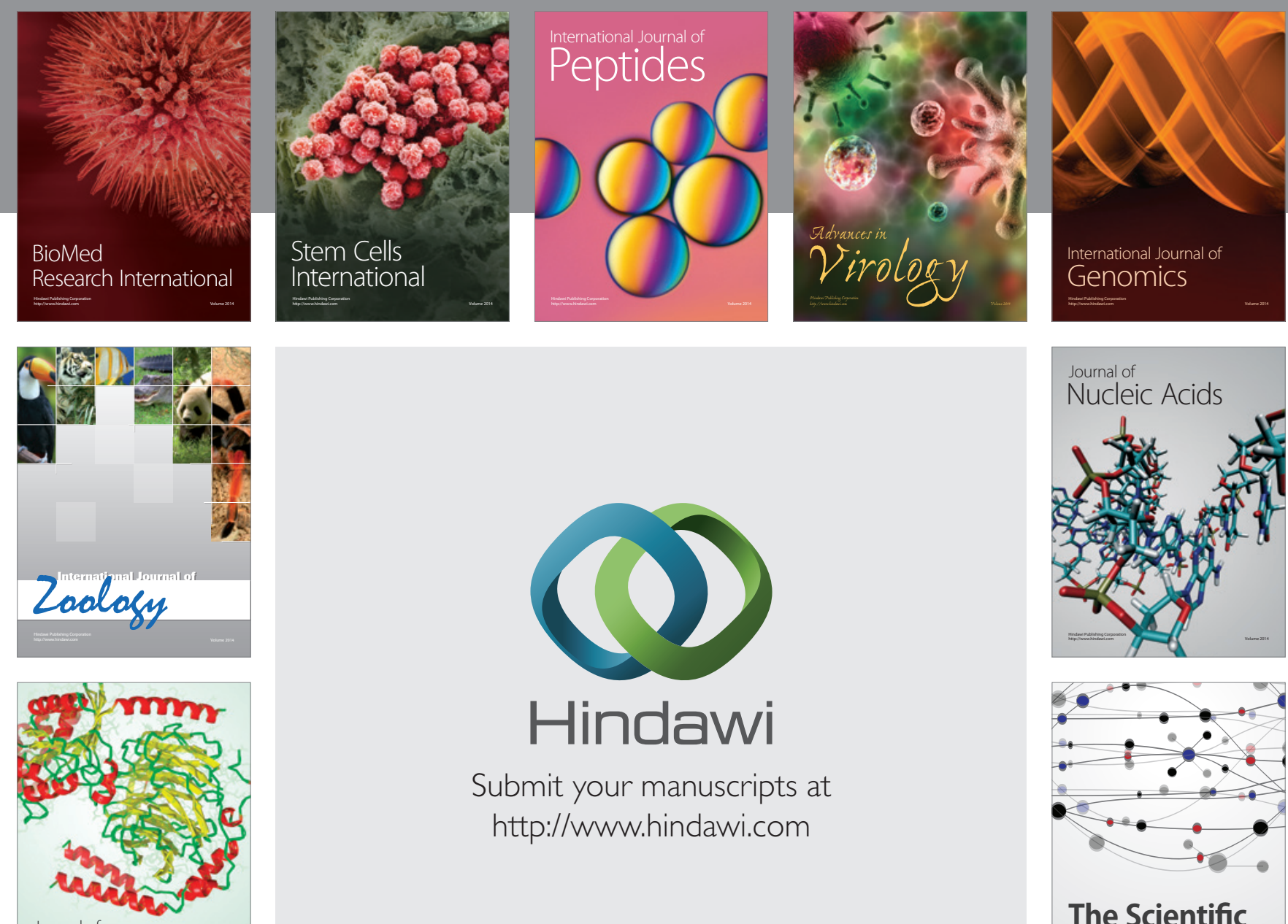

Submit your manuscripts at

http://www.hindawi.com

Journal of
Signal Transduction
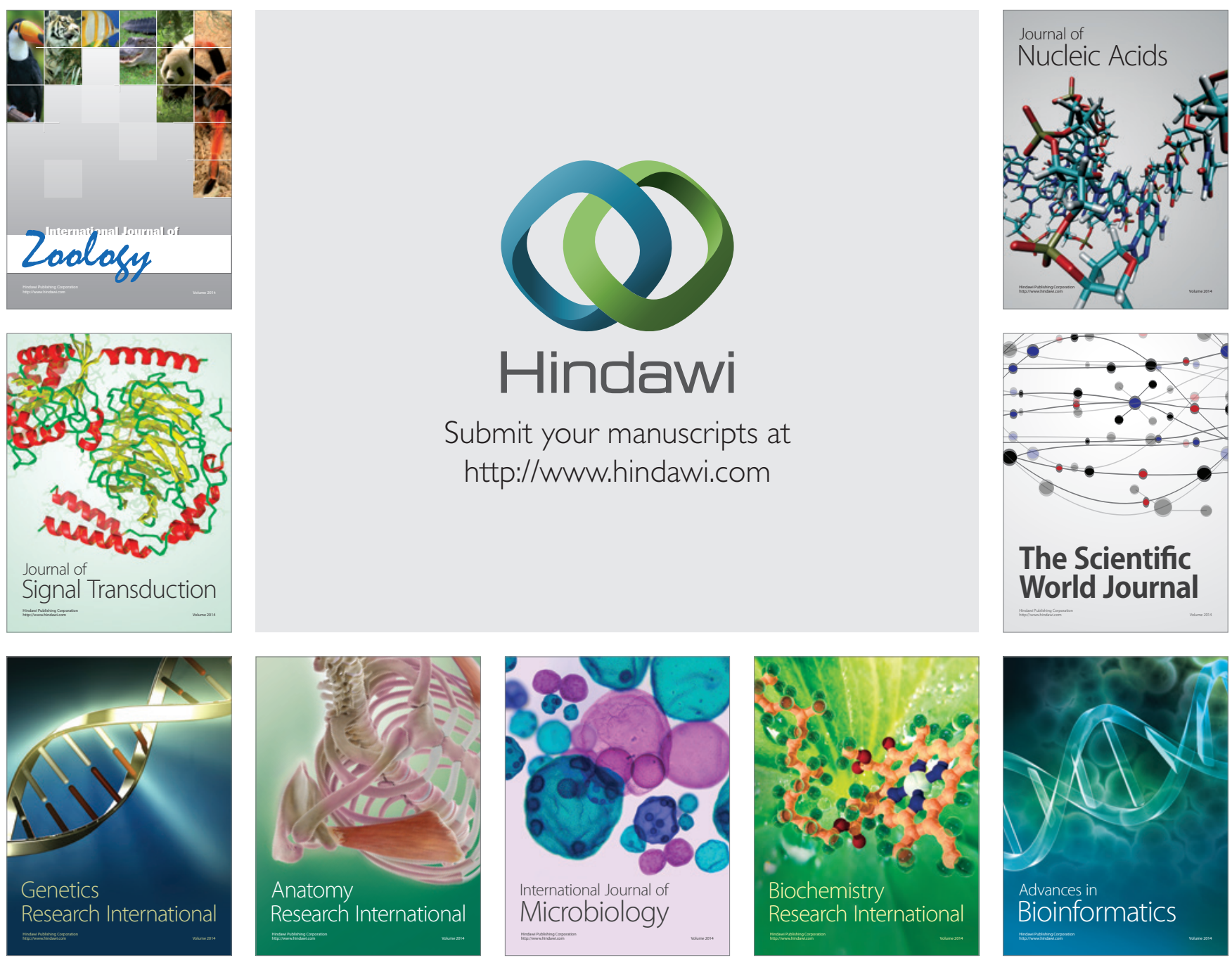

The Scientific World Journal
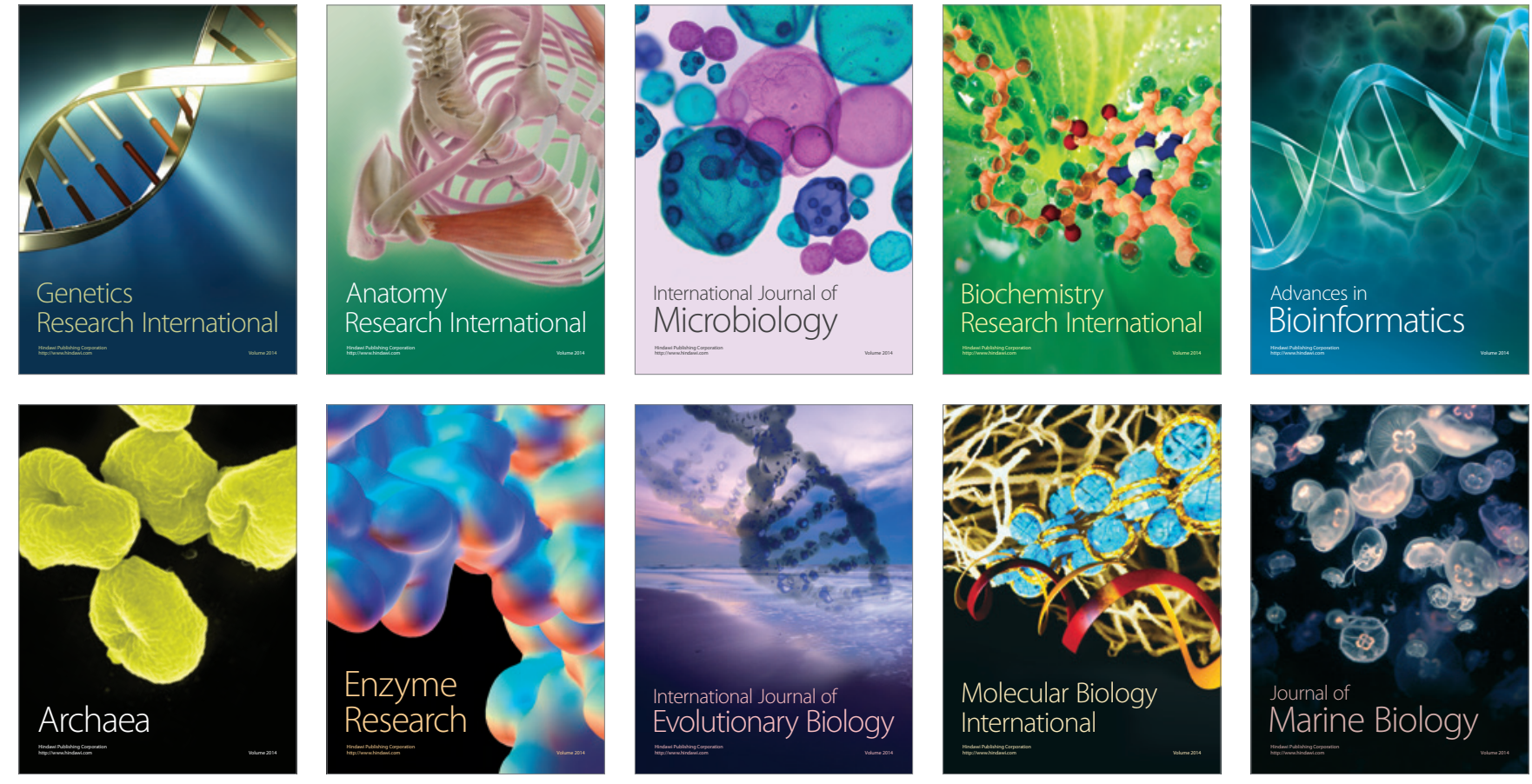